its degree exceeds 1,000 , while the formula $\frac{1}{3} n+1$ would place the upper limit of transitivity for such groups beyond 300 . These illustrations may suffice to exhibit clearly that a much smaller upper limit for the degree of transitivity of a primitive group which is neither alternating nor symmetric results from the use of the present theorem than the one given by $\frac{1}{3} n+1$, whenever $n$ is large. When $n=12=7+5$ the two theorems lead to the same upper limit. This is also true for the cases when $n$ is 8 or 9 . Since the groups whose degrees are less than 8 are so well known, it does not appear necessary to preserve the formula $\frac{1}{3} n+1$ as an upper limit of the degree of transitivity of substitution groups which do not include the alternating group, especially since the theorem proved above is based upon such very elementary considerations.

UNIVERSITY OF ILLINOIS.

\title{
THE PERMUTATIONS OF THE NATURAL NUMBERS CAN NOT BE WELL ORDERED.
}

\author{
BY PROFESSOR A. B. FRIZELL.
}

(Read before the American Mathematical Society, February 27, 1915.)

LET us tabulate the natural numbers according to the number of their prime factors, viz., the $n$th row shall consist of the products $\pi(\nu, n)$ of $n$ primes in order of magnitude. Form a new rectangular array wherein the $n$th column shall be composed of numbers from the $n$th row of the first scheme but arranged in rows by their column indices $\nu$ in the former, so that now the $i$ th row contains those products $\pi(\nu, n)$ for which $\nu$ is a product of $i$ primes. We obtain an infinite matrix of series

$3,5,11,17,31, \cdots ; 6,9,14,21,33, \cdots$; $12,18,27,30,50, \cdots ; 24,36,54,60,90, \cdots ; \cdots$ $7,13,23,29,43, \cdots ; 10,15,25,26,38, \cdots$; $20,28,44,45,66, \cdots ; 40,56,84,88,126, \cdots ; \cdots$ $19,37,61,71,103, \cdots ; 22,34,51,57,82, \cdots$; $42,52,76,92,116, \cdots ; 81,100,140,152,210, \cdots ; \cdots$ $53,89,151,173,251, \cdots ; 46,69,111,121,161, \cdots$; $70,105,154,171,236, \cdots ; 135,196,276,306,376, \cdots ; \cdots$

It is proposed to form permutations of the natural numbers 
by interchanges among the elements of this array and for the present purpose it is enough to consider only exchanges of elements in the same column and permutations of terms in the same element. For the purpose of this paper is to examine the consequences of the following

Assumption.-The permutations of an w-series can be well ordered,

and, denoting by $\Pi$ the ordinal type of this series of permutations, it is clear that a II-series may be obtained in either of the above ways, e. g., by permuting the terms in the first element or by permuting the elements in the first column. Indeed, if we select from the series of primes those whose numbers in the series are primes, the permutations of the whole set of natural numbers can be put into one-to-one correspondence with those obtained by only transposing pairs of consecutive members of the series

$$
3,5,11,17,31,41,59,67,83,109, \cdots
$$

i. e., $1,2,5,4,3,6, \cdots ; 1,2,3,4,11,6,7,8,9,10,5,12,13,14$, $15,16,31,18,19, \cdots$, etc. Another way of obtaining a II-series is by exchanging the first element of each column with the lower elements in the same column. It is easy to exhibit the one-to-one correspondence in this case by assigning to each $n$th digit $(i, n)$ in a given permutation of the natural numbers the interchange of the first with the $i$ th element in the $n$th column of our matrix. Thus to the permutation

$$
2,1,4,3,6,5,8,7, \cdots
$$

will correspond that obtained by raising to the first place the second, fourth, third, ... elements in the first, third, fourth, ... columns respectively, that is, the permutation

$1,2,7,4,13,6,3,8,9,10,23,70,5,14,15,16,29,105,19,20$, $21,22,11,81,25,26,154,28,17,171,43,32,33,34,35,100, \cdots$. Obviously the same reasoning would hold if in each column we should exchange the series of first terms with the other series of $n$th terms. Moreover, both sets of transpositions may be performed simultaneously and yield a II-series, since we get nothing but permutations of the natural numbers and clearly they are all different. In like manner, although we obtain a $\Pi$-series by permuting elements of a single column, we get no more by doing it in different columns independently. Similarly if we permute terms of a single element or make such permutations independently in different elements or if we combine this process with that of permuting elements in the 
same column, in each case the resulting set of permutations may be written as a $\Pi$-series.

We will now show that our assumption leads to a set of permutations of the natural numbers which is equivalent to the whole set but can not be written in a $\Pi$-series. In any well-ordered set every element is an $N$ th term in some $\omega$-series $(N=1,2, \cdots)$. For a given $N$ the set of all $N$ th terms shall be called the elements of the $N$ th kind. Permute independently the terms in different elements of our matrix in all possible ways and let $[P]$ denote the resulting set of permutations arranged in a $\Pi$-series.

There exists a permutation of the natural numbers which differs in every nth column of its matrix $(n=1,2, \cdots)$ from every element of the nth kind in $[P]$. The elements of the $n$th kind in $[P]$, being by hypothesis part of a well-ordered set, form also a well-ordered set $\left[P^{(n)}\right]$ of ordinal type $\Pi^{(n)} \leqq \Pi$. The proposition will be proved by establishing the following

Lемma. For each value of $n$ there exists a permutation of the natural numbers which differs in every Nth element in the nth column of its matrix from every element of the Nth kind in $\left[P^{(n)}\right]$.

It is not necessary actually to produce such permutations; it is sufficient to show that they exist. This is easily done by observing that the elements of the first kind in $\left[P^{(n)}\right]$ do not use up the $\Pi^{(n)}$-series of its totality and hence do not exhaust the $\Pi$-series of permutations available for the first element of the matrix. Hence there is a permutation of the natural numbers differing in the permutation performed on its first element from every element of the first kind in $\left[P^{(n)}\right]$ and at the same time different for the same reason from every element of the $N$ th kind in $\left[P^{(n)}\right]$ in the permutation performed on the $N$ th element $(N=2,3, \cdots)$ in the first column of its matrix. The same reasoning holds independently for every $n$th column $(n=2,3, \cdots)$ and, of course, there are in each case an infinity of such permutations $P^{\prime}$ outside of $[P]$. So we have, on the basis of our assumption, two sets, $[P]$ and $[P]+\left[P^{\prime}\right]$, which are both in one-to-one correspondence with the whole set of permutations of the natural numbers but can not be put into one-to-one correspondence with each other.

Whence the assumption is false.

McPherson, Kansas. 